\title{
Pupillary axis of a heterocentric astigmatic eye
}

\section{WF Harris}

\author{
Department of Optometry, University of Johannesburg, PO Box 524, Auckland Park, 2006 South \\ Africa
}

<wharris@uj.ac.za>

Received 25 July 2012; revised version accepted 3 February 2013

\begin{abstract}
The pupillary axis of the eye is a clinically useful concept usually defined as the line through the centre of the entrance pupil that is perpendicular to the cornea. However if the cornea is astigmatic then, strictly speaking, the entrance pupil is blurred and the pupillary axis is not well defined. A modified definition is offered in this paper: the pupillary axis is the infinite straight line containing the incident segment of the ray that passes through the centre of the (actual) pupil and is perpendicular to the first surface of the eye. The definition holds for the naked eye and for an eye with an implant in the anterior chamber. It also holds for the compound system of eye and optical instrument such as a contact lens in front of it if the first surface is interpreted as the first surface of the compound
\end{abstract}

system and the pupil as the limiting aperture of the compound system. Linear optics is applied to obtain a formula for the position and inclination of the pupillary axis at incidence onto the system; the refracting surfaces may be heterocentric and astigmatic. The formula allows one to examine the sensitivity of the pupillary axis to displacement of the pupil and any other changes in the anterior eye. Strictly the pupillary axis depends on the frequency of light but examples show that the dependence is probably negligible. The vectorized generalization of what is sometimes called angle lambda is easily calculated from the inclination of the pupillary axis and the line of sight. (S Afr Optom 2013 72(1) 3-10)

Key words: Pupillary axis, angle lambda, astigmatism, transference, linear optics

\section{Introduction}

The dictionary by Hofstetter and others ${ }^{1}$ defines the pupillary axis as "The line perpendicular to the cornea and passing through the centre of the entrance pupil of the eye". They go on to comment on its significance: "It is the line by means of which the direction of a person's gaze is objectively determined". Atchison and Smith ${ }^{2}$ define the pupillary axis in the same way. The pupillary axis, then, is a useful clinical concept. But how is it related to the structure of the eye and how is it affected by change in the eye? The purpose of this paper is to derive general formulae for the position and orientation of the pupillary axis in terms of the fundamental optical properties of the eye, the anterior part of the eye in particular. With these formulae one is able to locate the pupillary axis of an eye if the structure (curvatures, tilts, separations, indices of refraction, position of pupil centre) of the anterior part of the eye is known and determine the sensitivity of the axis to changes in any of these structures. We make use of linear optics. The refracting surfaces of the eye may be astigmatic and heterocentric. Because of the generality of the approach the results apply not only to the naked eye but also to the compound system of eye and optical device (for example, a contact lens) in front of it. 
Below we obtain explicit formulae for the position of the pupillary axis at incidence onto the eye and the inclination of the axis. We also obtain formulae for the transverse position of the axis at the pupil and the retina and for the transverse position and inclination of the ray at the retina whose incident segment is along the pupillary axis. Strictly the location of the pupillary axis depends on the frequency of light. We define the chromatic difference of the pupillary axes of an eye. The pupillary axis is compared with the line of sight of an eye and angle lambda determined from the difference between them. The mathematics is applied to the special case of a model eye with a single refracting surface. A numerical example is presented for a model eye with four separated tilted and nonaligned astigmatic refracting surfaces.

In the case of a model eye with a single refracting surface the pupillary axis is simply a straight line through the pupil centre and orthogonal to the surface. One expects the same to hold approximately for most eyes. The situation is more complicated, however, in the case of an eye with an implant in the anterior chamber or for the compound system of eye and optical device (a contact lens for example) in front of it. The approach here covers such situations.

\section{Definition of the pupillary axis}

Entrance and exit pupils are concepts of Gaussian optics. Because of astigmatism they do not generalize comfortably to linear optics. Being an image of the pupil in the anterior part of the eye each point in the pupil maps to an interval of Sturm if the cornea is astigmatic. Thus the entrance pupil is blurred. (It is also blurred because of chromatic dispersion.) The effect is usually small and may be negligible in the clinical environment but it means that the entrance pupil is not well defined. We, therefore, need a definition for pupillary axis which does not depend on the concept.

The centre of the entrance pupil is the (blurred) image of the centre of the actual pupil. Hence we can think of the pupillary axis in front of the eye as the segment of the ray from the centre of the pupil that leaves the eye orthogonal to the first surface of the eye. Of course we can reverse the direction of this ray. We, therefore, make the following definition: The pupillary axis of an eye is the straight line, infinite in both senses along it, which contains the incident segment of the ray that is both orthogonal to the first surface of the eye and through the centre of the actual pupil. We assign it a positive sense into the eye.

This definition is consistent with the definition mentioned above and has the advantage that it lends itself to analysis by means of linear optics. Strictly the first surface of the eye is the first surface of the tear film although for most purposes it can be taken as the first surface of the cornea. The definition generalizes to the compound system of eye and optical instrument (a contact lens for example) in front of it; the first surface of the eye becomes the first surface of the compound system and the pupil becomes the limiting aperture (usually the eye's pupil) of the compound system.

Figure 1 is a schematic representation of an eye and its pupillary axis. Two segments of a ray are shown (solid arrows): the first (the incident) segment is shown orthogonal to the first surface $\mathrm{K}$ of the eye and the second segment intersects the pupil at its centre P. No other refracting surfaces (back surface of the cornea and surfaces of the natural lens or of an implant) are shown. The pupillary axis, represented as a dashed line, is the infinite extrapolation, forward and backward, of the incident segment. Note that it does not intersect the pupil at its centre.

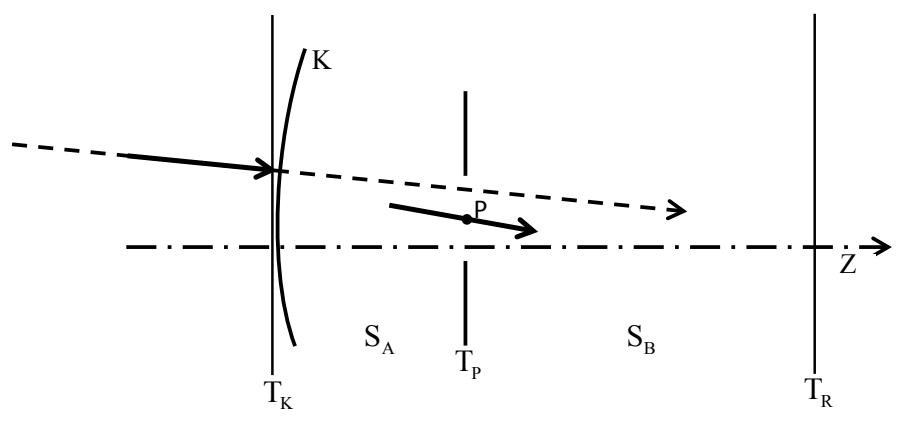

Figure 1 Schematic representation of the pupillary axis (dashed line) of an eye. The only components of the eye shown explicitly are the first refracting surface $\mathrm{K}$ of the eye and the iris. $\mathrm{Z}$ is the longitudinal axis. $\mathrm{S}_{\mathrm{A}}$ is the anterior part of the eye; it extends from entrance plane $T_{K}$ immediately in front of the tear film on the cornea to the plane of the pupil $T_{P}$. The posterior part of the eye $S_{B}$ extends from $T_{P}$ to transverse plane $T_{R}$ immediately in front of the retina. The two solid arrows are segments of the same ray traversing the eye, the first segment (the incident segment) is orthogonal to $\mathrm{K}$ and the second segment intersects the pupil in its centre P. 


\section{Locating the pupillary axis}

We make use of the symbolism used before ${ }^{3} . \mathrm{Z}$ is the longitudinal axis relative to which transverse position and inclinations are measured. System $\mathrm{S}$ is the whole eye from entrance plane $T_{K}$ immediately in front of the tear film of the cornea to exit plane $T_{R}$ immediately anterior to the retina. $T_{P}$ is the plane of the pupil; it partitions $\mathrm{S}$ into subsystems $\mathrm{S}_{\mathrm{A}}$ and $\mathrm{S}_{\mathrm{B}} \cdot \mathrm{S}_{\mathrm{A}}$ is the anterior portion of the eye or simply the anterior eye and similarly for $S_{B}$. Properties of $S_{A}$ are identified by means of subscript $A$ and similarly for $S_{B}$. The medium immediately anterior to $T_{K}$ has index of refraction $n_{0}$; the indices in the pupil and immediately anterior to the retina are $n_{\mathrm{P}}$ and $n_{\mathrm{R}}$ respectively. The centre of the pupil has transverse position represented by the vector $\mathbf{y}_{P}$.

The pupillary axis is fully defined if we can determine its inclination $\mathbf{a}_{\mathrm{K}}$ and its transverse position $\mathbf{y}_{\mathrm{K}}$ (both vectors) at incidence onto the eye, that is, at $\mathrm{T}_{\mathrm{K}}$. It is our objective, then, to obtain $\mathbf{a}_{\mathrm{K}}$ and $\mathbf{y}_{\mathrm{K}}$ in terms of fundamental properties of the eye.

From the definition we need to determine the incident segment of a ray that is (a) orthogonal to the first surface of the eye and (b) through the centre of the pupil. From linear optics we shall write equations in terms of $\mathbf{y}_{\mathrm{K}}$ and $\mathbf{a}_{\mathrm{K}}$ that represent these two aspects of the definition and then solve them for $\mathbf{y}_{\mathrm{K}}$ and $\mathbf{a}_{\mathrm{K}}$.

For aspect (a) the relevant optical system is the first surface of the eye. At normal incidence the ray is not deflected by the surface. It follows that, across the surface,

$\mathbf{C}_{\mathrm{K}} \mathbf{y}_{\mathrm{K}}+\mathbf{D}_{\mathrm{K}} \mathbf{a}_{\mathrm{K}} n_{0}+\boldsymbol{\pi}_{\mathrm{K}}=\mathbf{a}_{\mathrm{K}} n_{\mathrm{K}}$

where $n_{\mathrm{K}}$ is the index immediately posterior to the surface,

$\mathbf{C}_{\mathrm{K}}=\mathcal{K}_{\mathrm{K}}\left(n_{0}-n_{\mathrm{K}}\right)$

is the divergence of the surface,

$\mathbf{D}_{\mathrm{K}}=\mathbf{I}$

is its divarication,

$\boldsymbol{\pi}_{\mathrm{K}}=\mathbf{m}_{\mathrm{K}}\left(n_{0}-n_{\mathrm{K}}\right)$

is its deflectance, $\mathcal{K}_{\mathrm{K}}$ is its (matrix) curvature and $\mathbf{m}_{\mathrm{K}}$ its (vectorial) tilt. $\mathbf{I}$ is an identity matrix. Substituting from Equations 2 to 4 into Equation 1 and rearranging we find that

$$
\mathcal{K}_{\mathrm{K}} \mathbf{y}_{\mathrm{K}}+\mathbf{a}_{\mathrm{K}}=-\mathbf{m}_{\mathrm{K}} \text {. }
$$

Note that no indices of refraction remain in the equation.
For aspect (b) the system of interest is the anterior eye $\mathrm{S}_{\mathrm{A}}$. Across it linear optics allows us to write

$\mathbf{A}_{\mathrm{A}} \mathbf{y}_{\mathrm{K}}+\mathbf{B}_{\mathrm{A}} \mathbf{a}_{\mathrm{K}} n_{0}=\mathbf{y}_{\mathrm{P}}-\mathbf{e}_{\mathrm{A}}$.

Equations 5 and 6 are two simultaneous equations in $\mathbf{y}_{\mathrm{K}}$ and $\mathbf{a}_{\mathrm{K}}$ which represent the two aspects of the definition. They can be combined as

$$
\left(\begin{array}{cc}
\mathcal{K}_{\mathrm{K}} & \mathbf{I} \\
\mathbf{A}_{\mathrm{A}} & \mathbf{B}_{\mathrm{A}} n_{0}
\end{array}\right)\left(\begin{array}{l}
\mathbf{y}_{\mathrm{K}} \\
\mathbf{a}_{\mathrm{K}}
\end{array}\right)=\left(\begin{array}{c}
-\mathbf{m}_{\mathrm{K}} \\
\mathbf{y}_{\mathrm{P}}-\mathbf{e}_{\mathrm{A}}
\end{array}\right) .
$$

We write this equation as

$\mathbf{G x}_{\mathrm{K}}=\mathbf{d}$

where

$$
\begin{aligned}
& \mathbf{G}=\left(\begin{array}{ll}
\mathcal{K}_{\mathrm{K}} & \mathbf{I} \\
\mathbf{A}_{\mathrm{A}} & \mathbf{B}_{\mathrm{A}} n_{0}
\end{array}\right), \\
& \mathbf{x}_{\mathrm{K}}=\left(\begin{array}{l}
\mathbf{y}_{\mathrm{K}} \\
\mathbf{a}_{\mathrm{K}}
\end{array}\right)
\end{aligned}
$$

and

$\mathbf{d}=\left(\begin{array}{c}-\mathbf{m}_{\mathrm{K}} \\ \mathbf{y}_{\mathrm{P}}-\mathbf{e}_{\mathrm{A}}\end{array}\right)$.

We shall refer to $\mathbf{x}_{\mathrm{K}}$ as the incident location of the pupillary axis. $\mathbf{G}$ is the matrix coefficient and $\mathbf{d}$ the constant vector of the governing equation (Equation 8) of the pupillary axis.

Equation 8 is a linear equation in incident location $\mathbf{x}_{\mathrm{K}}$. Equations of this form are standard in linear algebra. We are interested in solutions $\mathbf{x}_{\mathrm{K}}$ to it. Depending on $\mathbf{G}$ and d there may be no solutions, one solution or an infinity of solutions. We shall consider only the usual case, that is, the case in which matrix coefficient $\mathbf{G}$ is nonsingular. Exceptional cases, that is, those in which $\mathbf{G}$ is singular, can be handled as described elsewhere ${ }^{4,5}$.

Provided $\mathbf{G}$ is nonsingular Equation 8 can be solved to give

$\mathbf{x}_{\mathrm{K}}=\mathbf{G}^{-1} \mathbf{d}$,

the incident location of the pupillary axis.

We note that the bottom block-rows of $\mathbf{G}$ and $\mathbf{d}$ are identical to the bottom block-rows of the corresponding matrices for the line of sight or sighting axis ${ }^{3}$.

Equation 12 enables one to calculate the incident location of the pupillary axis for an eye of known structure, as illustrated in the examples below. It fails, however, if the eye is such that its matrix coefficient $\mathbf{G}$ is singular. One can also anticipate potential computational problems when $\mathbf{G}$ is close to being singular.

A disadvantage of Equation 12 is that it gives little 
insight into relationships. More insight can be gained with the following alternative approaches although each brings with it additional potential problems of singularity and computational difficulty.

\section{Alternative approaches}

From Equation 5 we obtain

$\mathbf{a}_{\mathrm{K}}=-\mathcal{K}_{\mathrm{K}} \mathbf{y}_{\mathrm{K}}-\mathbf{m}_{\mathrm{K}}$

Substituting from this equation into Equation 6 and solving we obtain

$\mathbf{y}_{\mathrm{K}}=\left(\mathbf{A}_{\mathrm{A}}-\mathbf{B}_{\mathrm{A}} \mathcal{K}_{\mathrm{K}} n_{0}\right)^{-1}\left(\mathbf{y}_{\mathrm{P}}+\mathbf{B}_{\mathrm{A}} \mathbf{m}_{\mathrm{K}} n_{0}-\mathbf{e}_{\mathrm{A}}\right)$.

Finally substituting from Equation 14 into Equation 13 we find that

$\mathbf{a}_{\mathrm{K}}=-\mathcal{K}_{\mathrm{K}}\left(\mathbf{A}_{\mathrm{A}}-\mathbf{B}_{\mathrm{A}} \mathcal{K}_{\mathrm{K}} n_{0}\right)^{-1}\left(\mathbf{y}_{\mathrm{P}}+\mathbf{B}_{\mathrm{A}} \mathbf{m}_{\mathrm{K}} n_{0}-\mathbf{e}_{\mathrm{A}}\right)-\mathbf{m}_{\mathrm{K}} \cdot$

Equations 14 and 15 show that the changes in transverse position and inclination of the pupillary axis are linear in change in transverse position of the pupil centre, the proportionality matrices being $\left(\mathbf{A}_{\mathrm{A}}-\mathbf{B}_{\mathrm{A}} \mathcal{K}_{\mathrm{K}} n_{0}\right)^{-1}$ and $-\mathcal{K}_{\mathrm{K}}\left(\mathbf{A}_{\mathrm{A}}-\mathbf{B}_{\mathrm{A}} \mathcal{K}_{\mathrm{K}} n_{0}\right)^{-1}$ respectively. Of course these equations fail when $\mathbf{A}_{\mathrm{A}}-\mathbf{B}_{\mathrm{A}} \mathcal{K}_{\mathrm{K}} n_{0}$ is nonsingular.

One can also solve for $\mathbf{y}_{\mathrm{K}}$ and $\mathbf{a}_{\mathrm{K}}$ by first solving Equation 6 for $\mathbf{y}_{\mathrm{K}}$ and then substituting into Equation 5. These equations also have their potential problems of singularity and computational difficulty.

\section{Intersection of the pupillary axis with the pupil and the retina}

From the equations above we can calculate the transverse position at incidence and the inclination of the pupillary axis at incidence onto the first surface of the eye. It may be easier to visualize the axis in terms of the transverse position at incidence onto the first surface and at transverse plane $T_{P}$ or $T_{R}$. The transverse positions at the pupil and retina are

$\mathbf{y}_{\mathrm{KP}}=\mathbf{y}_{\mathrm{K}}+z_{\mathrm{A}} \mathbf{a}_{\mathrm{K}}$

and

$\mathbf{y}_{\mathrm{KR}}=\mathbf{y}_{\mathrm{K}}+z \mathbf{a}_{\mathrm{K}}$ respectively where $z_{\mathrm{A}}$ is the length of the anterior part of the eye (from $T_{P}$ to $T_{P}$ ) and $z$ is the length of the eye (from $T_{K}$ to $T_{R}$ ). Of course the inclination of the pupillary axis at the pupil and retina is simply $\mathbf{a}_{\mathrm{K}}$.

\section{Location at the retina of the ray defining the pupillary axis}

A ray incident onto the eye along the pupillary axis arrives at the retina at transverse position $\mathbf{y}_{R}$ and with inclination $\mathbf{a}_{\mathrm{R}} \cdot \mathbf{y}_{\mathrm{R}}$ and $\mathbf{a}_{\mathrm{R}}$ can be determined from

$$
\left(\begin{array}{c}
\mathbf{y}_{\mathrm{R}} \\
\mathbf{a}_{\mathrm{R}} n_{\mathrm{R}} \\
1
\end{array}\right)=\mathbf{T}\left(\begin{array}{c}
\mathbf{y}_{\mathrm{K}} \\
\mathbf{a}_{\mathrm{K}} n_{0} \\
1
\end{array}\right)
$$

where $\mathbf{T}$ is the transference of the whole eye.

\section{Chromatic difference of the pupillary axes}

The fundamental properties of an optical system usually depend on the frequency of the light involved. ${ }^{6}$ The bottom block-rows of $\mathbf{G}$ and $\mathbf{d}$ (Equations 9 and 11) depend on the top block-row of the transference of the anterior part of the eye. Hence the incident location of the pupillary axis depends on frequency. There is a pupillary axis for blue light (the blue pupillary axis) with incident location $\mathbf{x}_{\mathrm{K}}^{\mathrm{b}}$ and one for red light (the red pupillary axis) with location $\mathbf{x}_{\mathrm{K}}^{\mathrm{r}}$. (Here 'red' and 'blue' should be interpreted simply as labels for light of particular frequencies.) In keeping with what was done before $^{3}$ we define a chromatic difference of pupillary axes by means of

$$
\Delta \mathbf{x}_{\mathrm{K}}=\mathbf{x}_{\mathrm{K}}^{\mathrm{b}}-\mathbf{x}_{\mathrm{K}}^{\mathrm{r}} \text {. }
$$

In Example 1 below the chromatic difference of the pupillary axes is identically null and in Example 2 it turns out to be close to null.

\section{Difference between the line of sight and the pupillary axis}

A recent paper ${ }^{3}$ provides formulae for calculating the incident location of the line of sight or sighting axis of an eye. The difference between the pupillary and sighting axes is represented completely by the vector

$\left(\Delta \mathbf{x}_{\mathrm{K}}\right)_{\mathrm{PS}}=\left(\mathbf{x}_{\mathrm{K}}\right)_{\mathrm{SA}}-\left(\mathbf{x}_{\mathrm{K}}\right)_{\mathrm{PA}}$

where $\left(\mathbf{x}_{\mathrm{K}}\right)_{\mathrm{PA}}$ and $\left(\mathbf{x}_{\mathrm{K}}\right)_{\mathrm{SA}}$ represent the incident 
locations of the pupillary and sighting axes of an eye. The bottom two entries of $\left(\Delta \mathbf{x}_{\mathrm{K}}\right)_{\mathrm{PS}}$, that is, what we might represent by

$\left(\Delta \mathbf{a}_{\mathrm{K}}\right)_{\mathrm{PS}}=\left(\mathbf{a}_{\mathrm{K}}\right)_{\mathrm{SA}}-\left(\mathbf{a}_{\mathrm{K}}\right)_{\mathrm{PA}}$,

is the difference in inclination of the pupillary and sighting axes of the eye. It is a vectorial generalization of what is often called angle lambda ${ }^{2}$. The top two entries of $\left(\Delta \mathbf{x}_{\mathrm{K}}\right)_{\mathrm{PS}}$ represent the difference in incident transverse position,

$$
\left(\Delta \mathbf{y}_{\mathrm{K}}\right)_{\mathrm{PS}}=\left(\mathbf{y}_{\mathrm{K}}\right)_{\mathrm{SA}}-\left(\mathbf{y}_{\mathrm{K}}\right)_{\mathrm{PA}} \text {, }
$$

of the two axes.

\section{Example 1}

Let us examine a simple case in which the pupillary axis is obvious from the definition. We will then follow through the mathematics to confirm that the result does agree with what we know.

Consider what must be among the simplest possible examples one can think of: an 'eye' with a flat, tilted, single-surface 'cornea'. In Figure 2 the 'cornea' has tilt $\mathbf{m}_{\mathrm{K}}=\left(\begin{array}{c}0 \\ 0.01\end{array}\right)$, that is, 1 prism dioptre $(\mathrm{pd})$ about a horizontal axis (pantoscopic tilt). The tilt is positive because increase in the vertical coordinate implies increase in the horizontal coordinate. By the definition the pupillary axis is orthogonal to the 'cornea' and intersects the pupil at its centre: it is shown by means of the dashed line with negative inclination. It has inclination $\mathbf{a}_{\mathrm{K}}=\left(\begin{array}{c}0 \\ -0.01\end{array}\right)=-\mathbf{m}_{\mathrm{K}}$, that is, 1 pd downward. Its transverse position $\mathbf{y}_{\mathrm{K}}$ at incidence onto the 'eye' is above $\mathrm{P}$.

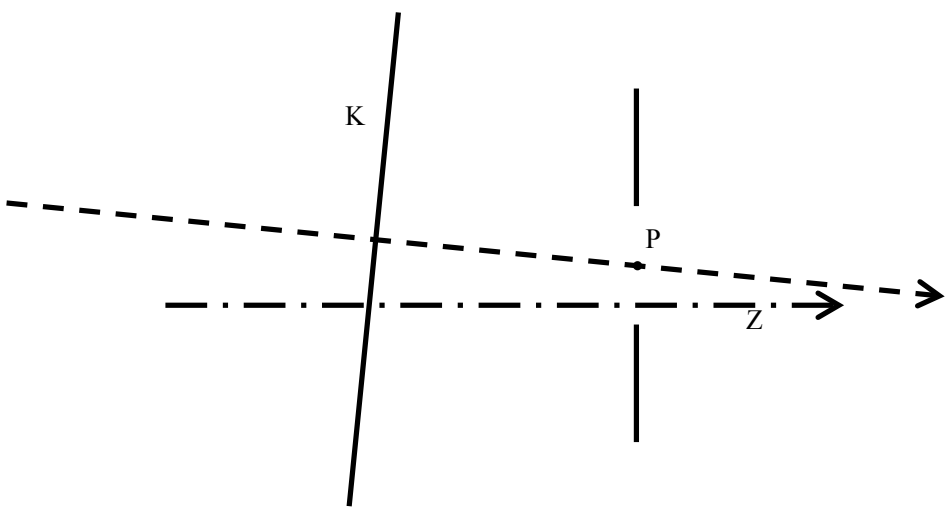

Figure 2 The pupillary axis (dashed) of the 'eye' with a flat tilted single-surface 'cornea' treated in Example 1.
We turn now to the mathematics. The anterior portion of the 'eye' consists of a single flat refracting surface $\left(\mathcal{K}_{\mathrm{K}}=\mathbf{O}\right.$, where $\mathbf{O}$ is the $2 \times 2$ null matrix $)$ with tilt $\mathbf{m}_{\mathrm{K}}$ followed by a homogeneous gap of width $z_{\mathrm{A}}$ and index $n_{\mathrm{A}}$. The centre of the pupil has transverse position $\mathbf{y}_{\mathrm{P}}$. The transference of the anterior eye is

$$
\begin{aligned}
\mathbf{T}_{\mathrm{A}} & =\left(\begin{array}{ccc}
\mathbf{I} & \mathbf{I} z_{\mathrm{A}} / n_{\mathrm{A}} & \mathbf{0} \\
\mathbf{O} & \mathbf{I} & \mathbf{0} \\
\mathbf{o}^{\mathrm{T}} & \mathbf{0}^{\mathrm{T}} & 1
\end{array}\right)\left(\begin{array}{ccc}
\mathbf{I} & \mathbf{O} & \mathbf{0} \\
\mathbf{O} & \mathbf{I} & \mathbf{m}_{\mathrm{K}}\left(n_{0}-n_{\mathrm{A}}\right) \\
\mathbf{o}^{\mathrm{T}} & \mathbf{0}^{\mathrm{T}} & 1
\end{array}\right) \\
& =\left(\begin{array}{ccc}
\mathbf{I} & \mathbf{I} z_{\mathrm{A}} / n_{\mathrm{A}} & \mathbf{m}_{\mathrm{K}} z_{\mathrm{A}}\left(n_{0}-n_{\mathrm{A}}\right) / n_{\mathrm{A}} \\
\mathbf{O} & \mathbf{I} & \mathbf{m}_{\mathrm{K}}\left(n_{0}-n_{\mathrm{A}}\right) \\
\mathbf{o}^{\mathrm{T}} & \mathbf{o}^{\mathrm{T}} & 1
\end{array}\right) .
\end{aligned}
$$

o is the $2 \times 1$ null matrix. Hence

$$
\mathbf{G}=\left(\begin{array}{cc}
\mathbf{O} & \mathbf{I} \\
\mathbf{I} & \mathbf{I} z_{\mathrm{A}} n_{0} / n_{\mathrm{A}}
\end{array}\right)
$$

and

$\mathbf{d}=\left(\begin{array}{c}-\mathbf{m}_{\mathrm{K}} \\ \mathbf{y}_{\mathrm{P}}-\mathbf{m}_{\mathrm{K}} z_{\mathrm{A}}\left(n_{0}-n_{\mathrm{A}}\right) / n_{\mathrm{A}}\end{array}\right)$.

As is readily confirmed by multiplication

$\mathbf{G}^{-1}=\left(\begin{array}{cc}-\mathbf{I} z_{\mathrm{A}} n_{0} / n_{\mathrm{A}} & \mathbf{I} \\ \mathbf{I} & \mathbf{O}\end{array}\right)$.

Hence we find that the incident location of the pupillary axis is

$$
\mathbf{x}_{\mathrm{K}}=\left(\begin{array}{c}
\mathbf{m}_{\mathrm{K}} z_{\mathrm{A}}+\mathbf{y}_{\mathrm{P}} \\
-\mathbf{m}_{\mathrm{K}}
\end{array}\right),
$$

a result in agreement with our expectations.

It is clear from the entries on the right of Equation 23 that the incident location of the pupillary axis is independent of frequency. It follows that the chromatic difference of pupillary axes (Equation 19) is identically null in this case.

\section{Example 2}

Consider the model eye with parameters listed in Table 1. It is the same model treated before ${ }^{3,4}$. There are four separated astigmatic tilted refracting surfaces. The principal meridians of the refracting surfaces are not aligned. The eye is in air $\left(n_{0}=1\right)$ and has length $z=24.5 \mathrm{~mm}$. The anterior eye has length $z_{\mathrm{A}}=24.5 \mathrm{~mm}$. The centre of the pupil is located $1 \mathrm{~mm}$ to the left of axis $Z$. Hence we set 
$\mathbf{y}_{\mathrm{P}}=\left(\begin{array}{c}-1 \\ 0\end{array}\right) \mathrm{mm}$.

The centre of the fovea has transverse position $\mathbf{y}_{\mathrm{R}}=\left(\begin{array}{c}\cos 45^{\circ} \\ \sin 45^{\circ}\end{array}\right) \mathrm{mm}$ which is required for locating the sighting axis. We assume that the centre of the pupil is the pupillary sighting centre and that the centre of the fovea is the retinal sighting centre. We consider light of frequencies corresponding to the wavelengths in vacuum of 486.1 ('blue') and 656.3 ('red') nm and use the equations for refractive index presented by Villegas and coworkers ${ }^{7}$.

Table 1 Principal radii of curvature, separation, and tilt of surfaces of model eye used in the numerical example. K1 and K2 represent the first and second surfaces of the cornea and L1 and L2 the first and second surfaces of the lens.

\begin{tabular}{|c|c|c|c|c|}
\hline Surface & $\begin{array}{l}\text { Principal radii } \\
\mathrm{mm}\{\text { degr }\} \mathrm{mm}\end{array}$ & $\begin{array}{l}\text { Separation } \\
\mathrm{mm}\end{array}$ & Tilt & \\
\hline \multirow[t]{2}{*}{ K1 } & $5.8\{180\} 7$ & & $(0.06$ & $-0.05)^{\mathrm{T}}$ \\
\hline & & 0.5 & & \\
\hline \multirow[t]{2}{*}{$\mathrm{K} 2$} & $5\{10\} 6.2$ & & $(0.04$ & $0.06)^{\mathrm{T}}$ \\
\hline & & 4 & & \\
\hline \multirow[t]{2}{*}{$\mathrm{L} 1$} & $4.1\{20\} 5$ & & $(-0.07$ & $0.1)^{\mathrm{T}}$ \\
\hline & & 4 & & \\
\hline \multirow[t]{2}{*}{ L2 } & $-5\{70\}-6.2$ & & $(-0.05$ & $-0.03)^{\mathrm{T}}$ \\
\hline & & 16 & & \\
\hline
\end{tabular}

From Table 1 the curvature and tilt of the first surface are

$\mathcal{K}_{\mathrm{K}}=\left(\begin{array}{cc}1 / 5.8 & 0 \\ 0 & 1 / 7\end{array}\right) \mathrm{mm}^{-1}$

and

$\mathbf{m}_{\mathrm{K}}=\left(\begin{array}{c}0.06 \\ -0.05\end{array}\right)$

respectively. From Equations 9 and 11 we obtain the red matrix coefficient and vector constant

$\mathbf{G}^{\mathrm{r}}=\left(\begin{array}{cccc}0.1724 \mathrm{~mm}^{-1} & 0 \mathrm{~mm}^{-1} & 1 & 0 \\ 0 \mathrm{~mm}^{-1} & 0.1429 \mathrm{~mm}^{-1} & 0 & 1 \\ 0.8066 & 0.0008 & 3.3717 \mathrm{~mm} & 0.0003 \mathrm{~mm} \\ 0.0008 & 0.8396 & 0.0003 \mathrm{~mm} & 3.3701 \mathrm{~mm}\end{array}\right)$

and

$\mathbf{d}^{\mathrm{r}}=\left(\begin{array}{c}-0.06 \\ 0.05 \\ -0.9292 \mathrm{~mm} \\ -0.0703 \mathrm{~mm}\end{array}\right)$
The bottom two rows of these two matrices were calculated previously ${ }^{3}$. The blue matrices $\mathbf{G}^{\mathrm{b}}$ and $\mathbf{d}^{\mathrm{b}}$ are identical except that the numbers in the bottom two rows are

$\left(\begin{array}{llll}0.8035 & 0.0008 & 3.3538 & 0.0003 \\ 0.0008 & 0.8370 & 0.0003 & 3.3522\end{array}\right)$

and

$\left(\begin{array}{l}-0.9281 \\ -0.0712\end{array}\right)$

respectively.

Applying Equation 12 we obtain the incident location of the red pupillary axis:

$\mathbf{x}_{\mathrm{K}}^{\mathrm{r}}=\left(\begin{array}{c}-3.2247 \mathrm{~mm} \\ -0.6602 \mathrm{~mm} \\ 0.4960 \\ 0.1443\end{array}\right)$

The incident transverse position is some $3.3 \mathrm{~mm}$ to the left of and a little below $Z$. The axis has an inclination to the right and up at just under $30^{\circ}$ from $\mathrm{Z}$. Because these 
numbers are probably larger than one could consider paraxial one should regard them as no more than rough estimates.

The incident location $\mathbf{x}_{\mathrm{K}}^{\mathrm{b}}$ of the blue pupillary axis is identical to $\mathbf{x}_{\mathrm{K}}^{\mathrm{r}}$ rounded to the same number of decimal places. The chromatic difference of the pupillary axes is (Equation 19)

$$
\Delta \mathbf{x}_{\mathrm{K}}=\left(\begin{array}{c}
-7.9 \mathrm{~nm} \\
7.4 \mathrm{~nm} \\
1.4 \times 10^{-6} \\
-1.1 \times 10^{-6}
\end{array}\right)
$$

which surely means that, in effect, the red and blue pupillary axes are indistinguishable at incidence.

The incident location of the red sighting axis of this eye was previously ${ }^{3}$ found to be

$$
\left(\mathbf{x}_{\mathrm{K}}\right)_{\mathrm{SA}}=\left(\begin{array}{c}
-1.2896 \mathrm{~mm} \\
-0.1956 \mathrm{~mm} \\
0.0330 \\
0.0282
\end{array}\right) \text {. }
$$

Hence, from Equation 20

$$
\left(\Delta \mathbf{x}_{\mathrm{K}}\right)_{\mathrm{PS}}=\left(\begin{array}{c}
1.9351 \mathrm{~mm} \\
0.4646 \mathrm{~mm} \\
-0.4630 \\
-0.1161
\end{array}\right)
$$

Thus the generalized red angle lambda is (Equation 21)

$$
\left(\Delta \mathbf{a}_{\mathrm{K}}\right)_{\mathrm{PS}}=\left(\begin{array}{l}
-0.4630 \\
-0.1161
\end{array}\right),
$$

that is about $26^{\circ}$ to the left and $7^{\circ}$ down.

The pupillary axis intersects the plane of the pupil $T_{P}$ and the retina $T_{R}$ at points with transverse positions (Equation 16)

$\mathbf{y}_{\mathrm{KP}}=\left(\begin{array}{l}-0.993 \\ -0.011\end{array}\right) \mathrm{mm}$,

that is, not quite at the centre of the pupil, and (Equation 17)

$\mathbf{y}_{\mathrm{KR}}=\left(\begin{array}{l}8.927 \\ 2.875\end{array}\right) \mathrm{mm}$

respectively. There is no difference for red and blue light for the number of significant digits shown.

The pupillary axis is sketched in Figure 3 where the view is along longitudinal axis $\mathrm{Z}$ and into the eye. The small circles locate the intersection of the pupillary axis with the first surface of the cornea, the pupil and the retina.

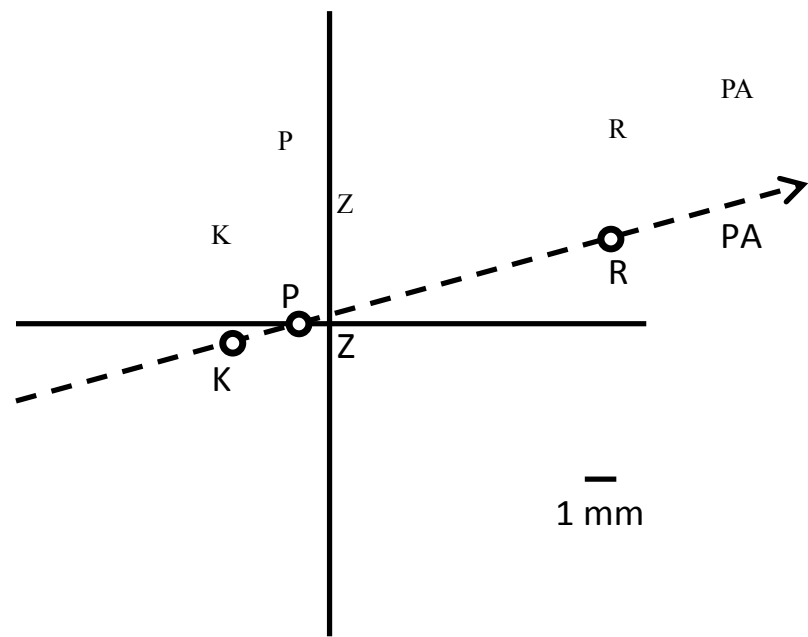

Figure 3 The pupillary axis (PA) for the model eye in Example 2 showing the intersections with the first surface of the cornea $(\mathrm{K})$, the pupil $(\mathrm{P})$ and the retina $(\mathrm{R})$. The view is into the eye along longitudinal axis $\mathrm{Z}$.

\section{Concluding remarks}

The pupillary axis of an eye has been defined here as the infinite straight line that contains the incident segment of the ray which is perpendicular to the first surface of the eye and goes through the centre of the actual pupil. This definition is consistent with the traditional clinical definition of the pupillary axis as the straight line perpendicular to the cornea and through the centre of the entrance pupil. It has the advantage of being amenable to analysis by means of linear optics. Using linear optics we have derived an equation (Equation 12) for the location of the pupillary axis, that is, the inclination of the axis and its transverse position at incidence onto the eye. A numerical example (Example 2) illustrates the use of the equation in the case of a fairly extreme model eye; the results probably exceed paraxial limits and should not be interpreted too literally in this case. Alternative equations (Equations 14 and 15) give more insight into relations and display the dependence of the pupillary axis on the position of the pupil centre explicitly.

One expects the equations to work satisfactorily in most situations of interest in practice. However there are circumstances in which Equation 12 breaks down; for example an eye with a cornea that is a spherical shell both of whose surfaces have centres at the centre of the 
pupil. In such a case there is an infinity of pupillary axes; every normal defines a pupillary axis. Such an eye would have a cornea of exceptionally high relative curvature. There are also eyes for which the centre of the pupil is not easy to define.

Strictly the pupillary axis depends on the frequency of light but calculations suggest that the dependence is weak and probably clinically negligible.

The difference between the inclinations of the pupillary and the sighting axes gives the vectorial generalization of angle lambda; it is also calculated for the model eye in Example 2.

\section{Acknowledgements}

I gratefully acknowledge a grant from the National Research Foundation. I thank T Evans and $\mathrm{RD}$ van Gool for continuing discussions.

\section{References}

1. Hofstetter HW, Griffin JR, Berman MS, Everson RW. Dictionary of Visual Science and Related Clinical Terms. 5th ed. Butterworth-Heinemann: Boston, 2000 p. 306.

2. Atchison DA, Smith G. Optics of the Human Eye. Butterworth-Heinemann: Oxford, 2000 pp. 31-36.

3. Harris WF, van Gool RD, T Evans. Line of sight of a heterocentric astigmatic eye. Ophthal Physiol Opt 201333 57-66.

4. Harris WF. Achromatic axes and their linear optics. Vis Res 201258 1-9.

5. Harris WF. Optical axes of eyes and other optical systems Optom Vis Sci 200986 537-541.

6. Evans T, Harris WF. Dependence of the transference of a reduced eye on frequency of light. S Afr Optom 201170 149-155.

7. Villegas ER, Carretero L, Fimia A. Le Grand eye for the study of ocular chromatic aberration. Ophthal Physiol Opt $199616528-531$. 